\title{
ON G-FIBERINGS OVER THE CIRCLE WITHIN A COBORDISM CLASS
}

\author{
TAMIO HARA
}

\section{Introduction}

Conner and Floyd [1] have characterized those unoriented cobordism classes that admit a representative which fibers over the circle $S^{1}$. They have shown that a closed manifold $M$ is cobordant to a bundle over $S^{1}$ if and only if $\chi(M) \equiv 0(\bmod 2)$, where $\chi$ is the Euler characteristic. Let $G$ be a finite abelian group of odd order and $\mathfrak{\Re}_{*}^{G}$ the cobordism group of unoriented closed $G$ manifolds. The purpose of this paper is to determine when a class $\beta$ in $\mathfrak{N}_{*}^{G}$ has a representative which fibers equivariantly over $S^{1}$ such that the action of $G$ takes place within fiber. The author [3] has discussed such a question in case where $G=Z_{2^{r}}$, the cyclic group of order $2^{r}$.

In Section 1, we first introduce an SK group $S K_{*}^{G}$ resulting from equivariant cuttings and pastings ( $G$-SK processes) of closed $G$-manifolds. The abbreviation SK stands for Schneiden und Kleben in German. Kosniowski [7] has obtained some generators of $S K_{*}^{G}$ as a free $S K_{*}$-module, where $S K_{*}$ is an SK ring of closed manifolds in Karras, Kreck, Neumann and Ossa [5] (Proposition 1.4). As an example, we perform $G$-SK processes on some complex projective space with $G$ action and write it by the above generators (Example 1.8).

In Section 2, we consider a notion of $G$-SK invariant studied in [5] and [7]. Let $T$ be a map for closed $G$-manifolds which takes values in the ring $\boldsymbol{Z}$ of rational integers and is additive with respect to the disjoint union of $G$-manifolds. Such a $T$ is said to be a $G$-SK invariant if it is invariant under $G$-SK processes. Given a $G$-manifold $M$, let $M_{\sigma}$ be a $G$-submanifold of $M$ consisting of those points whose slice types containing $\sigma$. Then a map $\chi_{\sigma}$ defined by $\chi_{\sigma}(M)=$ $\chi\left(M_{\sigma}\right)$ is a $G$-SK invariant. Further, for a subgroup $H$ of $G$, the map $\chi^{H}$ defined by $\chi^{H}(M)=\chi\left(M^{H}\right)$ is also a $G$-SK invariant, where $M^{H}=\{x \in M \mid$ $h x=x$ for any $h \in H\}$. We see that $\chi^{H}=\sum_{\sigma} \chi_{\sigma}$ summing over all $\sigma$ with $H$ as an isotropy subgroup. The above $T$ is considered to be an additive homomorphism $T: S K_{*}^{G} \rightarrow \boldsymbol{Z}$. We determine a form of $T$ by using those $\chi_{\sigma}$ and have a base for a $Z$-module $\mathscr{T}_{*}^{G}$ consisting of all $G$-SK invariants (Theorem 2.6).

In Section 3, we devote to a study of $G$-fiberings over $S^{1}$. Let $\overline{S K}_{*}^{G}$ be $S K_{*}^{G}$

1991 Mathematics Subject Classification: 57S17.

Keywords: $G$-manifold, cutting and pasting, SK group, SK invariant, cobordism, $G$-fibering. Received May 14, 2002; revised July 9, 2003. 
factored by the equivariant cobordism relation. Let $\overline{\mathscr{T}}_{*}^{G}$ be a $\boldsymbol{Z}_{2}$-vector space consisting of all homomorphisms $\bar{T}: \overline{S K}_{*}^{G} \rightarrow \boldsymbol{Z}_{2}$. Such a map $\bar{T}$ is called a $G$ $\overline{S K}$ invariant, namely a $G$-SK invariant (modulo 2 ) and simultaneously invariant under equivariant cobordism. We first show that a $G$-SK invariant $T$ which is considered to take values in $\boldsymbol{Z}_{2}$ via the surjection $\boldsymbol{Z} \rightarrow \boldsymbol{Z}_{2}$, is always a $G-\overline{S K}$ invariants (Theorem 3.8). The kernel $F_{*}^{G}$ of the natural surjection $j_{*}: \mathfrak{N}_{*}^{G} \rightarrow$ $\overline{S K}_{*}^{G}$ is exactly generated by those classes, each of which admits a representative fibered equivariantly over $S^{1}$. We characterize the elements of $F_{*}^{G}$ by using $G$ $\overline{S K}$ invariants (Theorem 3.10 and Proposition 3.12). Finally, in case $G=Z_{7}$, we give a non-zero element of $F_{*}^{G}$ by using the complex projective space with $G$ action treated in Example 1.8 (Example 3.14).

\section{Equivariant cutting and pasting}

Let $G$ be a finite abelian group. In this paper, a $G$-manifold means an unoriented compact smooth manifold together with a smooth action of $G$. Let $N_{i}(i=1,2)$ be $m$-dimensional $G$-manifolds and $\phi, \psi: \partial N_{1} \rightarrow \partial N_{2}$ equivariant diffeomorphisms. Pasting along their boundaries, we have closed $G$-manifolds $M_{1}=N_{1} \cup_{\phi} N_{2}$ and $M_{2}=N_{1} \cup \cup_{\psi}$. Then it is said that $M_{1}$ and $M_{2}$ are obtained from each other by an equivariant cutting and pasting ( $G$-SK process) [5, 7]. Let $\mathscr{M}_{m}^{G}$ be the set of all $m$-dimensional closed $G$-manifolds. Then it is an abelian semigroup with respect to the disjoint union + and has a zero given by the empty set $\emptyset$.

Definition 1.1. $G$ manifolds $M_{1}$ and $M_{2} \in \mathscr{M}_{m}^{G}$ are said to be $G$-SK equivalent, in symbols $M_{1} \sim M_{2}$, if there is a $G$ manifold $K \in \mathscr{M}_{m}^{G}$ such that $M_{1}+K$ and $M_{2}+K$ can be obtained from each other by a finite sequence of equivariant cuttings and pastings.

The $G$-SK equivalence $\sim$ is an equivalence relation on the set $\mathscr{M}_{m}^{G}$ and the set $\Gamma_{m}^{G}=\mathscr{M}_{m}^{G} / \sim$ of all equivalence classes is a cancellative abelian semigroup. Let denote by $[M]$ the class containing a $G$-manifold $M$. Denote by $S K_{m}^{G}$ the Grothendieck group of $\Gamma_{m}^{G}$. We then have a graded $S K_{*}$-module $S K_{*}^{G}=$ $\oplus_{m \geq 0} S K_{m}^{G}$ given by the cartesian product of manifolds. Here $S K_{*}$ is an SK ring of closed manifolds which is a polynomial ring over $\boldsymbol{Z}$ with a generator $\alpha$ represented by the real projective plane $\boldsymbol{R} P^{2}$ [7; Theorem 2.5.1 (i)].

We assume for the remainder of this paper that $G$ is an abelian group of odd order. A $G$-module means a finite-dimensional real vector space together with a linear action of $G$. For a subgroup $H$ of $G$, let $C(H)$ consist of all subgroups $J$ of $H$ such that the quotient $H / J \cong \boldsymbol{Z}_{d}$, a cyclic group of odd order $d$. Then, for $J \in C(H)$ an irreducible $H$-module $V(J, j)$ is defined as follows: if $d=1$ then $V(H, 1)=\boldsymbol{R}$ with the trivial action of $H$, while if $d \geq 3$ then $V(J, j)$ is the set $\mathbf{C}$ of complex numbers with a generator $h$ of $H / J$ acting by multiplication by $\exp \left(2 \pi i m_{j} / d\right)$, where $\left\{m_{j}\right\}$ is the complete set of integers such that $0<m_{1}<$ $m_{2}<\cdots<m_{\varphi(d)}<d$ and each $m_{j}$ is prime to $d$ ( $\varphi$, the Euler phi function). If $M$ 
is a $G$-manifold and $x \in M$, then there is a $G_{x}$-module $U_{x}$ which is equivariantly diffeomorphic to a $G_{x}$-neighbourhood of $x$. Here $G_{x}=\{g \in G \mid g x=x\}$ is the isotropy subgroup at $x$. The module $U_{x}$ decomposes as $U_{x}=\boldsymbol{R}^{p} \oplus V_{x}$, where $G_{x}$ acts trivially on $\boldsymbol{R}^{p}$ and $V_{x}^{G_{x}}=\{0\}$. We refer to the pair $\sigma_{x}=\left[G_{x} ; V_{x}\right]$ as a slice type of $x$. By a $G$-slice type in general, we mean a pair $\sigma=[H ; V]$ of a subgroup $H$ and an $H$-module $V$ with $V^{H}=\{0\}$. More precisely, $V$ is a product of non-trivial irreducible $H$-modules $V(J, j)\left(J \in C(H)\right.$ with $H / J \cong \boldsymbol{Z}_{d}$ and $1 \leq j<\frac{1}{2} \varphi(d)+1$ ) (cf. [7; Theorem 1.6.1]). We denote by $\sigma_{0}$ the slice type $[\{1\} ;\{0\}]$, where $\{1\}$ is the trivial group. Let $S t(G)$ be the set of all $G$-slice types. There is a partial ordering on $\operatorname{St}(G)$ such that $[H ; V] \preceq[K ; W]$ if $[H ; V]$ is a slice type of $G$-manifold $G \times{ }_{K} W$. Further, we give a total ordering on $S t(G)$, which preserves the one $\preceq$, as follows. For any positive divisor $k$ of $|G|$, let $L(k)$ be the set consisting of all subgroups $H$ of $G$ such that $|H|=k$. First order the elements in $L(k)$ appropriately, then this ordering gives the one $<$ on the set of all subgroups of $G$, preserving inclusion of subgroups, that is, if $H \subseteq K$ then $H \leq K$. Moreover, for any $H$ such an ordering leads to the one on the set of non-trivial irreducible $H$-modules: $\quad V\left(J_{1}, j_{1}\right)<V\left(J_{2}, j_{2}\right)$ if $J_{2}<J_{1}$ or $J_{1}=J_{2}$ and $j_{1}<j_{2}$. Finally we order the elements in $S t(G)$ as follows:

(1) $[H ; V]<[K ; W]$ if $\operatorname{dim}(V)<\operatorname{dim}(W)$.

(2) Suppose that $\operatorname{dim}(V)=\operatorname{dim}(W)$, then $[H ; V]<[K ; W]$ if $H<K$.

(3) Suppose that $\operatorname{dim}(V)=\operatorname{dim}(W)$ and $H=K$, then $[H ; V]<[H ; W]$ if $V<W$ in the ordering of $H$-modules induced lexicographically from the one of irreducible $H$-modules (cf. [7; Section 1.7]).

Definition 1.2. Let $W$ be a $K$-module and $H$ a subgroup of $K$. Then denote by $W_{H}$ an $H$-module $W$ induced from $H \subseteq K$. Let $\left\{W_{k}\right\}$ be the set of all non-trivial irreducible $K$-modules. If $\tau=[K ; W], W=\prod_{k} W_{k}^{a(k)}(a(k) \geq 0)$ is a slice type, then we define a slice type $\tau_{H}$ by $\tau_{H}=[H ; V]$, where $V$ is the nontrivial part of the $H$-module $\prod_{k}\left(W_{k}\right)_{H}^{a(k)}$. Since $\left(W_{k}\right)_{\{1\}}=\boldsymbol{R}^{2}$, we have that $\tau_{\{1\}}=\sigma_{0}$ for any $\tau$. Let $|\tau|=\operatorname{dim}(W)$ be the dimension of $\tau$.

Remark 1.3. (i) More precisely, let $W_{k}=V(L, j)$ for some $L \subset K$ with $K / L \simeq \boldsymbol{Z}_{a}$ and the integer $m_{j}$ such that $0<m_{j}<a,\left(m_{j}, a\right)=1$. Then $\left(W_{k}\right)_{H}=$ $V\left(L \cap H, j^{\prime}\right)$ with $0<m_{j^{\prime}}<b, \quad\left(m_{j^{\prime}}, b\right)=1$, where $H /(L \cap H)=L H / L \simeq \boldsymbol{Z}_{b}$. The integer $j^{\prime}$ is determined by the action $L H / L$ on $\left(W_{k}\right)_{H}$ induced from the one of $K / L$ on $W_{k}$. We see that $\left(W_{k}\right)_{H}$ is the trivial $H$-module $\boldsymbol{R}^{2}$ only if $H \subseteq L$. It follows that the difference $|\tau|-\left|\tau_{H}\right|$ is the sum of $\operatorname{dim}\left(\left(W_{k}\right)_{H}\right) \quad(=2)$ with $H \subseteq L$.

(ii) $W_{H}=\boldsymbol{R}^{|\tau|-\left|\tau_{H}\right|} \times V$ as an $H$-module and $W^{H}=\left(W_{H}\right)^{H}=\boldsymbol{R}^{|\tau|-\left|\tau_{H}\right|} \times\{0\}$ has slice types $\tau_{U}(H \subseteq U \subseteq K)$ as a $K$-invariant subspace of $W$. Note that $\tau_{U} \leq \tau$ because $\left|\tau_{U}\right| \leq|\tau|$.

Proposition 1.4 (cf. [7; Theorem 5.2.1]). $S K_{*}^{G}$ is a free $S K_{*}$-module with basis $\mathscr{B}=\{y[\sigma] ; \sigma=[H ; V] \in S t(G)\}$, where $y[\sigma]=\left[G \times_{H} \boldsymbol{R} P(V \times \boldsymbol{R})\right]$ and $\boldsymbol{R} P(V \times \boldsymbol{R})$ denotes the real projective space of the product $V \times \boldsymbol{R}$. 
Now, by using the total ordering on $S t(G)$, we rename the $G$-slice types: $\sigma_{0}=\rho_{0}, \rho_{1}, \rho_{2}, \ldots$ with the condition that if $i<j$ then $\rho_{i}<\rho_{j}$. Set $\mathscr{F}_{k}=\left\{\rho_{j}\right.$; $j \leq k\}$, then $\mathscr{F}_{k}$ is a family of $G$-slice types in the sense of that in [7; Section 1.2].

COROLlaRY 1.5. If a G-manifold $M$ has slice types $\sigma_{x} \in \mathscr{F}_{k}(x \in M)$, then the class $[M]$ is a linear combination over $S K_{*}$ by the elements $y\left[\rho_{j}\right]$ with $\rho_{j} \in \mathscr{F}_{k}$.

Lemma 1.6. For G-modules $U_{i}(i=1,2)$, let $S\left(U_{1} \times U_{2}\right)$ be a $G \times S^{1}$-sphere, that is the G-sphere together with the natural action of the circle group $S^{1}$. Then there is an SK equivalence:

$$
2 S\left(U_{1} \times U_{2}\right) \stackrel{\left(S^{1}\right)}{\sim} S\left(U_{1} \times \boldsymbol{R}\right) \times S\left(U_{2}\right)+S\left(U_{1}\right) \times S\left(U_{2} \times \boldsymbol{R}\right),
$$

where we use a symbol $\stackrel{\left(S^{1}\right)}{\sim}$ instead of $\sim$ because the above G-SK process is compatible with the action of $S^{1}$.

Proof. Let $N_{1}=N_{2}=S\left(U_{1}\right) \times D\left(U_{2}\right)+D\left(U_{1}\right) \times S\left(U_{2}\right)$, where $S\left(U_{i}\right)$ and the disk $D\left(U_{i}\right)$ are considered to be $G \times S^{1}$-spaces. Then we obtain the above equivalence by pasting $\partial N_{1}$ to $\partial N_{2}$ by the natural $G \times S^{1}$-equivariant identifications $\phi$ and $\psi$.

Lemma 1.7. For G-modules $V_{i}$ such that $V_{i}^{G}=\{0\} \quad(i=1,2)$, we have the following $S K$ equivalences.

(i) $S\left(\boldsymbol{R}^{2 k+1} \times V_{1}\right) \sim 2 \boldsymbol{R} P^{2 k} \times \boldsymbol{R} P\left(V_{1} \times \boldsymbol{R}\right)$.

(ii) $\boldsymbol{R} P\left(V_{1} \times \boldsymbol{R}\right) \times \boldsymbol{R} P\left(V_{2} \times \boldsymbol{R}\right) \sim \boldsymbol{R} P\left(V_{1} V_{2} \times \boldsymbol{R}\right)$.

Proof. We first consider (i). Let $S K_{*}^{G}(p t, p t)$ be an SK group resulting from cuttings and pastings of $G$-manifolds with boundary in $[2,4]$. It follows that $\left[D\left(V_{1}\right)\right]=\left[\boldsymbol{R} P\left(V_{1} \times \boldsymbol{R}\right)\right]$ in $S K_{*}^{G}(p t, p t)$ since $V_{1}$ is a product of twodimensional irreducible $G$-modules (cf. [4; Lemma 3.8 and Example 3.9 (3.3)]). Hence we obtain the equivalence in case $k=0:\left[S\left(V_{1} \times \boldsymbol{R}\right)\right]=2\left[\boldsymbol{R} P\left(V_{1} \times \boldsymbol{R}\right)\right]$ by making use of the map $\mathscr{D}_{*}: S K_{*}^{G}(p t, p t) \rightarrow S K_{*}^{G}$ given by $\mathscr{D}_{*}([M])=[M \cup M]$, the double of a $G$-manifold $M$. Further, when $k \geq 1$, set $\left(U_{1}, U_{2}\right)=\left(\boldsymbol{R}^{2 k+1}, V_{1}\right)$, forgetting $S^{1}$-action, in the equivalence in Lemma 1.6. Then

$$
2 S\left(\boldsymbol{R}^{2 k+1} \times V_{1}\right) \sim P_{1}+P_{2},
$$

where $P_{1}=S^{2 k+1} \times S\left(V_{1}\right)$ and $P_{2}=S^{2 k} \times S\left(V_{1} \times \boldsymbol{R}\right)$. Since $S^{2 k+1} \sim \emptyset$ and $S^{2 k} \sim 2 \boldsymbol{R} P^{2 k}$, we have that $2 S\left(\boldsymbol{R}^{2 k+1} \times V_{1}\right) \sim P_{2} \sim 2 \boldsymbol{R} P^{2 k} \times 2 \boldsymbol{R} P\left(V_{1} \times \boldsymbol{R}\right)$ (cf. [7; Theorem 2.5.1 (ii)]). Thus (i) follows since $S K_{*}^{G}$ has no torsion (cf. Proposition 1.4). Next we prove (ii). Let $\left(U_{1}, U_{2}\right)=\left(V_{1}, V_{2} \times \boldsymbol{R}\right)$, then $2 S\left(V_{1} V_{2} \times \boldsymbol{R}\right) \sim$ $S\left(V_{1} \times \boldsymbol{R}\right) \times S\left(V_{2} \times \boldsymbol{R}\right)+S\left(V_{1}\right) \times S\left(V_{2} \times \boldsymbol{R}^{2}\right)$ by Lemma 1.6. It is seen that $S\left(V_{1}\right)$ and $S\left(V_{2} \times \boldsymbol{R}^{2}\right) \sim \emptyset$ since they are odd-dimensional $G$-manifolds (cf. Proposition 1.4). Hence $4 \boldsymbol{R} P\left(V_{1} V_{2} \times \boldsymbol{R}\right) \sim 2 \boldsymbol{R} P\left(V_{1} \times \boldsymbol{R}\right) \times 2 \boldsymbol{R} P\left(V_{2} \times \boldsymbol{R}\right)$ by (i), which implies the result. 
Example 1.8. Consider the case where $G=Z_{p}$ ( $p$; odd prime). The nontrivial irreducible $G$-modules are $V_{j}=C$ with a generator of $G$ acting by multiplication by $\exp (2 \pi i j / p) \quad\left(1 \leq j \leq t=\frac{1}{2}(p-1)\right)$. We denote by $\langle a(1)$, $a(2), \ldots, a(t)\rangle$ a slice type $\sigma=[G ; V]$ with $V=\prod_{1 \leq j \leq t} V_{j}^{a(j)}$. Let $M=$ $C P\left(C^{a(0)} \times \sigma\right)$ be the associated complex projective space of the product $C^{a(0)} \times V$ with $a(0) \geq 0$. Then $[M]$ is represented by the generators of $S K_{*}^{G}$ in Proposition 1.4 as

$$
[M]=\sum_{0 \leq k \leq t} a(k) \alpha^{a(k)-1} y\left[\sigma_{(k)}\right],
$$

where $\sigma_{(k)}=\sigma$ if $k=0$,

$$
\begin{array}{r}
\langle a(k-1)+a(k+1), a(k-2)+a(k+2), \ldots, \\
a(0)+a(2 k), a(2 k+1), \ldots, a(t), 0, \ldots, 0\rangle
\end{array}
$$

if $1 \leq k<\frac{1}{2} t$

$$
\begin{aligned}
& \langle a(k-1)+a(k+1), a(k-2)+a(k+2), \ldots, \\
& \quad a(2 k-t)+a(t), a(2 k-t-1), \ldots, a(0), 0, \ldots, 0\rangle
\end{aligned}
$$

if $\frac{1}{2} t \leq k<t$ or

$$
\langle a(t-1), a(t-2), \ldots, a(0)\rangle
$$

if $k=t$. To show (1.8.1), we use the relation in Lemma 1.6. Set $\left(U_{1}, U_{2}\right)=$ $\left(V_{0}^{a(0)}, V\right)$, where $V_{0}=C$ with the natural $S^{1}$-action. Then

$$
2 S\left(V_{0}^{a(0)} \times V\right) \stackrel{\left(S^{1}\right)}{\sim} S\left(V_{0}^{a(0)} \times \boldsymbol{R}\right) \times S(V)+S\left(V_{0}^{a(0)}\right) \times S(V \times \boldsymbol{R}) .
$$

Next divide $V$ as $V=V_{1}^{a(1)} \times V^{\prime}$ with $V^{\prime}=\prod_{2 \leq j \leq t} V_{j}^{a(j)}$ and put $\left(U_{1}, U_{2}\right)=$ $\left(V_{1}^{a(1)}, V^{\prime}\right)$. Then

$$
2 S(V) \stackrel{\left(S^{1}\right)}{\sim} S\left(V_{1}^{a(1)} \times \boldsymbol{R}\right) \times S\left(V^{\prime}\right)+S\left(V_{1}^{a(1)}\right) \times S\left(V^{\prime} \times \boldsymbol{R}\right) .
$$

Taking this to $(1.8 .2)$, we have

$$
\begin{aligned}
2^{2} S\left(V_{0}^{a(0)} \times V\right) \stackrel{\left(S^{1}\right)}{\sim} & S\left(V_{0}^{a(0)} \times \boldsymbol{R}\right) \times S\left(V_{1}^{a(1)} \times \boldsymbol{R}\right) \times S\left(V^{\prime}\right) \\
+ & S\left(V_{0}^{a(0)} \times \boldsymbol{R}\right) \times S\left(V_{1}^{a(1)}\right) \times S\left(V^{\prime} \times \boldsymbol{R}\right) \\
+ & 2 S\left(V_{0}^{a(0)}\right) \times S(V \times \boldsymbol{R}) .
\end{aligned}
$$

Continuing such an SK process on $S\left(V^{\prime}\right)$ inductively, we have

$$
2^{t} S\left(V_{0}^{a(0)} \times V\right) \stackrel{\left(S^{1}\right)}{\sim} P+\sum_{0 \leq k<t} 2^{t-1-k} P_{k},
$$

where 


$$
\begin{aligned}
P & =\left(\prod_{0 \leq j<t} S\left(V_{j}^{a(j)} \times \boldsymbol{R}\right)\right) \times S\left(V_{t}^{a(t)}\right), \\
P_{k} & =\left(\prod_{0 \leq j<k} S\left(V_{j}^{a(j)} \times \boldsymbol{R}\right)\right) \times S\left(V_{k}^{a(k)}\right) \times S\left(\prod_{k<j \leq t} V_{j}^{a(j)} \times \boldsymbol{R}\right) .
\end{aligned}
$$

This induces an SK equivalence on the orbit spaces with respect to $S^{1}$ :

$$
2^{t} C P\left(V_{0}^{a(0)} \times V\right) \sim \bar{P}+\sum_{0 \leq k<t} 2^{t-1-k} \overline{P_{k}} .
$$

Here, it follows from Lemma 1.7 that $\bar{P}$ fibers equivariantly over $\overline{S\left(V_{t}^{a(t)}\right)}=$ $C P^{a(t)-1}$ with fiber

$$
\begin{aligned}
F & =\prod_{0 \leq j<t} S\left(\left(V_{j} \otimes V_{t}\right)^{a(j)} \times \boldsymbol{R}\right) \sim 2^{t} \boldsymbol{R} P\left(\prod_{0 \leq j<t}\left(V_{j} \otimes V_{t}\right)^{a(j)} \times \boldsymbol{R}\right) \\
& =2^{t} \boldsymbol{R} P\left(\sigma_{(t)} \times \boldsymbol{R}\right)
\end{aligned}
$$

and $\overline{P_{k}}$ fibers equivariantly over $\overline{S\left(V_{k}^{a(k)}\right)}=C P^{a(k)-1}$ with fiber

$$
\begin{aligned}
F_{k} & =\left(\prod_{0 \leq j<k} S\left(\left(V_{j} \otimes V_{k}\right)^{a(j)} \times \boldsymbol{R}\right)\right) \times S\left(\prod_{k<j \leq t}\left(V_{j} \otimes V_{k}\right)^{a(j)} \times \boldsymbol{R}\right) \\
& \sim 2^{k} \prod_{0 \leq j<k} \boldsymbol{R} P\left(\left(V_{j} \otimes V_{k}\right)^{a(j)} \times \boldsymbol{R}\right) \times 2 \boldsymbol{R} P\left(\prod_{k<j \leq t}\left(V_{j} \otimes V_{k}\right)^{a(j)} \times \boldsymbol{R}\right) \\
& \sim 2^{k+1} \boldsymbol{R} P\left(\prod_{j \neq k}\left(V_{j} \otimes V_{k}\right)^{a(j)} \times \boldsymbol{R}\right)=2^{k+1} \boldsymbol{R} P\left(\sigma_{(k)} \times \boldsymbol{R}\right) .
\end{aligned}
$$

From these, we have $\bar{P} \sim C P^{a(t)-1} \times F$ and $\overline{P_{k}} \sim C P^{a(k)-1} \times F_{k}(0 \leq k<t)$ (cf. [7; Theorem 2.4.1 (iv)]). It is seen that $\left[C P^{a(k)-1}\right]=a(k) \alpha^{a(k)-1}$ in $S K_{*}$ since $\chi\left(C P^{a(k)-1}\right)=a(k)$ (cf. [7; Theorem 2.5.1 (ii)]). Therefore we obtain the desired equality by taking (1.8.4) and (1.8.5) in (1.8.3).

Remark 1.9. In case of $G=\boldsymbol{Z}_{2^{r}}$, we have obtained a similar equality as (1.8.1) by performing an SK process on $G$-manifolds with boundary (cf. [2; Example 2.12 (ii)]).

\section{2. $G$-SK invariants}

In this section, we determine a form of $G$-SK invariants.

Definition 2.1. Let $\sigma=[H ; V] \in S t(G)$ and $M$ a $G$-manifold. Then define 
$M_{\sigma}$ to be the set consisting of those points $x \in M$ such that $\left(\sigma_{x}\right)_{H}=\sigma$ in the sense of Definition 1.2.

Remark 2.2. Let $M_{H}$ be $M$ with the induced action of $H$, then $M_{\sigma}$ is precisely the set $\left(M_{H}\right)_{\sigma}=\left\{x \in M_{H} ; \sigma_{x}=\sigma\right\}$. Since $\sigma$ is maximal in the family $\mathscr{F}\left(M_{H}\right)=\left\{\sigma_{x} ; x \in M_{H}\right\}$ with respect to the partial ordering $\preceq$ given in Section 1, $M_{\sigma}$ is a $G$-invariant submanifold of $M$ with $\operatorname{dim}\left(M_{\sigma}\right)=\operatorname{dim}(M)-|\sigma|$ by the slice theorem (cf. [5; Chapter 3]). In case $\sigma=\sigma_{0}$, we have that $M_{\sigma_{0}}=M$. The submanifold $M^{H}$ of $M$ decomposes as $M^{H}=\sum_{\sigma} M_{\sigma}$ summing over all $\sigma$ with $H$ as an isotropy subgroup.

Example 2.3. For $\tau=[K ; W] \in S t(G)$, let $M=G \times{ }_{K} \boldsymbol{R} P(W \times \boldsymbol{R})$ be a representative of the class $y[\tau]$ in $\mathscr{B}$ (cf. Proposition 1.4). The slice types of $M$ are the same as those of $G \times \times_{K}(W \times \boldsymbol{R})$ (or $W$ ) because $W$ is a complex $K$ module. If $H$ is a subgroup of $K$, then $M_{H}=G / K \times \boldsymbol{R} P\left(W_{H} \times \boldsymbol{R}\right)$ with the induced action of $H$ given by $h(([g],[v, t]))=([g],[h v, t])$ for $h \in H$ and $([g],[v, t]) \in$ $M_{H}$. On the other hand, if $H$ is not a subgroup of $K$, then $M^{H}=\emptyset$. Hence it follows that $\left[M_{\sigma}\right]=|G / K|\left[\boldsymbol{R} P^{|\tau|-\left|\tau_{H}\right|}\right]=|G / K| \alpha^{\left(|\tau|-\left|\tau_{H}\right|\right) / 2}$ if $\sigma=\tau_{H}$ with $H \subseteq K$ or $\left[M_{\sigma}\right]=0$ otherwise (cf. Remark 1.3 (ii) and [7; Theorem 1.7.1, Remark 1.7.2]). We see that $\left[\boldsymbol{R} P^{2 m}\right]=\alpha^{m}$ in $S K_{2 m}$ by considering the SK process as in Lemma 1.7 (ii) when $\left(V_{1}, V_{2}\right)=\left(\boldsymbol{C}, \boldsymbol{C}^{m-1}\right)$ (cf. [7; Theorem 2.5.1]).

Definition 2.4. Let $T: \mathscr{M}_{m}^{G} \rightarrow \boldsymbol{Z}$ be an additive map, that is, if $M=$ $M_{1}+M_{2}$ then $T(M)=T\left(M_{1}\right)+T\left(M_{2}\right)$. We call $T$ a $G$-SK invariant or simply an invariant if $T\left(N_{1} \cup_{\phi} N_{2}\right)=T\left(N_{1} \cup_{\psi} N_{2}\right)$ for any $G$-diffeomorphisms $\phi$ and $\psi: \partial N_{1} \rightarrow \partial N_{2}$ in Section 1. If $M_{1} \sim M_{2}$, then $T\left(M_{1}\right)=T\left(M_{2}\right)$. Thus the map $T$ induces an additive homomorphism $T: S K_{m}^{G} \rightarrow \boldsymbol{Z}$. The set $\mathscr{T}_{m}^{G}$ consisting of all these invariants is a $\boldsymbol{Z}$-module under the natural addition.

Example 2.5. Given a slice type $\sigma \in S t(G)$, let $\chi_{\sigma}$ be a map defined by $\chi_{\sigma}(M)=\chi\left(M_{\sigma}\right)$ for any $G$-manifold $M$. Then it is an invariant since $M \sim M^{\prime}$ implies $M_{\sigma} \sim M_{\sigma}^{\prime}$ naturally. Note that $\chi_{\sigma_{0}}=\chi$ since $M_{\sigma_{0}}=M$. Further, for any subgroup $H$ of $G$, the map $\chi^{H}$ defined by $\chi^{H}(M)=\chi\left(M^{H}\right)$ is also an invariant and the equality $\chi^{H}=\sum_{\sigma} \chi_{\sigma}$ holds in $\mathscr{T}_{m}^{G}$ (cf. Remark 2.2).

Let $H$ be a subgroup of $G$. Then, by using the total ordering on $\operatorname{St}(G)$, define inductively integers $n_{H}(K)$ for subgroups $K$ with $H \subseteq K \subseteq G$ as follows:

$$
n_{H}(H)=1, \quad n_{H}(K)=|K / H|-\sum_{H \subseteq L \subset K} n_{H}(L),
$$

where $L \subset K$ means that $L \subseteq K$ but $L \neq K$. If $H=\{1\}$, then the integers $n_{\{1\}}(K)$ coincide with those $n_{i}$ in [6; Definition 5.3]. For $\sigma=[H ; V] \in S t(G)$ and a subgroup $K$ with $H \subset K$, denote by $\mathscr{S}_{K}(\sigma)$ the set consisting of those slice types $\tau=[K ; W]$ such that $\tau_{H}=\sigma$. 
TheOREM 2.6. For $\sigma=[H ; V] \in \operatorname{St}(G)$, define $\theta_{\sigma}$ by

$$
\theta_{\sigma}=|G / H|^{-1}\left\{\chi_{\sigma}+\sum_{H \subset K \subseteq G} n_{H}(K)\left(\sum_{\tau \in \mathscr{S}_{K}(\sigma)} \chi_{\tau}\right)\right\} .
$$

Then the set $\left\{\theta_{\sigma} ;|\sigma| \leq 2 n\right\}$ provides a basis for $\mathscr{T}_{2 n}^{G}$ as a free $\boldsymbol{Z}$-module. On the other hand, $\mathscr{T}_{2 n+1}^{G}=\{0\}$.

Proof. First we see that $\mathscr{T}_{2 n+1}^{G}=\{0\}$ because $S K_{2 n+1}^{G}=\{0\}$ by Proposition 1.4. For $\sigma=[H ; V]$ with $|\sigma| \leq 2 n$, let $g_{\sigma}: S K_{2 n}^{G} \rightarrow S K_{2 n-|\sigma|}$ be a map given by $g_{\sigma}([M])=\left[M_{\sigma}\right]$ and $f_{\sigma}$ a map defined by

$$
f_{\sigma}=|G / H|^{-1}\left\{g_{\sigma}+\sum_{H \subset K \subseteq G} n_{H}(K)\left(\sum_{\tau \in \mathscr{S}_{K}(\sigma)} \alpha^{(|\tau|-|\sigma|) / 2} g_{\tau}\right)\right\} \text {. }
$$

Now look at the basis elements of $\mathscr{B}$ in Proposition 1.4. Then, given $\mu=$ $[K ; W] \in S t(G)$ the values $f_{\sigma}(y[\mu])$ which do not vanish are $f_{\mu_{L}}(y[\mu])=\alpha^{\left(|\mu|-\left|\mu_{L}\right|\right) / 2}$ $(L \subseteq K)$. In fact, if $\sigma=\mu_{L}$ for some $L(\subseteq K)$, then

$$
\begin{aligned}
f_{\mu_{L}}(y[\mu]) & =|G / L|^{-1}\left\{g_{\mu_{L}}(y[\mu])+\sum_{L \subset U \subseteq K} n_{L}(U) \alpha^{\left(\left|\mu_{U}\right|-\left|\mu_{L}\right|\right) / 2} g_{\mu_{U}}(y[\mu])\right\} \\
& =|K / L|^{-1}\left(\sum_{L \subseteq U \subseteq K} n_{L}(U)\right) \alpha^{\left(|\mu|-\left|\mu_{L}\right|\right) / 2} \\
& =\alpha^{\left(|\mu|-\left|\mu_{L}\right|\right) / 2}
\end{aligned}
$$

by Example 2.3 and the equality $\sum_{L \subseteq U \subseteq K} n_{L}(U)=|K / L|$. On the other hand, if $\sigma \notin\left\{\mu_{L} ; L \subseteq K\right\}$, then $\mu_{U} \notin \mathscr{S}_{U}(\sigma)$ for $U \subseteq K$. This implies that $g_{\sigma}(y[\mu])=g_{\tau}(y[\mu])=0$ in (2.6.1) and $f_{\sigma}(y[\mu])=0$ (cf. Example 2.3). Therefore each $f_{\sigma}$ induces an $S K_{*}$-homomorphism $f_{\sigma}: S K_{2 *}^{G}=\sum_{n} S K_{2 n}^{G} \rightarrow S K_{2 *-|\sigma|}=$ $\sum_{n \geq(1 / 2)|\sigma|} S K_{2 n-|\sigma|}$ of degree $-|\sigma|$. Now we recall the ordering of $G$-slice types: $\sigma_{0}=\rho_{0}, \rho_{1}, \rho_{2}, \ldots$ with the condition that if $i<j$ then $\rho_{i}<\rho_{j}$. This ordering ensure that if $\mu=[K ; W]$ then $\mu_{L}<\mu$ for $L \subset K$. Let us define an $S K_{*^{-}}$ homomorphism $f_{*}$ by

$$
f_{*}=\oplus_{k} f_{\rho_{k}}: S K_{2 *}^{G} \rightarrow A=\oplus_{k} S K_{2 *-\left|\rho_{k}\right|},
$$

where $f_{\rho_{k}}\left(y\left[\rho_{k}\right]\right)=[p t]_{k}$, the generator of $S K_{2 *-\left|\rho_{k}\right|} \cong S K_{*}$ as an $S K_{*}$-module. We can totally order the basis elements of $\mathscr{B}=\left\{y\left[\rho_{k}\right] ; k \geq 0\right\}$ and $\mathscr{B}^{\prime}=\left\{[p t]_{k} ; k \geq 0\right\}$ for $A$ naturally. Then it follows from (2.6.2) that $f_{*}$ is isomorphic because the matrix relative to the ordered bases $\mathscr{B}$ and $\mathscr{B}^{\prime}$ is triangular with components 1 on the diagonal. Now let $T$ be an element of $\mathscr{T}_{2 n}^{G}$, then there is a factorization

$$
T: S K_{2 n}^{G} \stackrel{f_{*}}{\cong} \oplus_{k} S K_{2 n-\left|\rho_{k}\right|} \stackrel{\oplus_{k} \chi}{\cong} \oplus_{k} \boldsymbol{Z} \stackrel{T^{\prime}}{\rightarrow} \boldsymbol{Z}
$$


for some $T^{\prime}$, where the direct sum is taken over all $k$ with $\left|\rho_{k}\right| \leq 2 n$ (cf. [7; Theorem 2.5.1 (ii)]). This implies that $T=\sum_{k} T^{\prime}\left(1_{k}\right) \theta_{\rho_{k}}$, where $\theta_{\rho_{k}}=\chi \circ f_{\rho_{k}}$ and $1_{k}=1$ in the $k$-th copy of $\boldsymbol{Z}$ in $\oplus_{k} \boldsymbol{Z}$. Note that $\left\{\rho_{k} ;\left|\rho_{k}\right| \leq 2 n\right\}=$ $\{\sigma ;|\sigma| \leq 2 n\}$ because the ordering on $\operatorname{St}(G)$ preserves the dimension $|\sigma|$. Thus the set $\left\{\theta_{\sigma} ;|\sigma| \leq 2 n\right\}$ provides a basis for $\mathscr{T}_{2 n}^{G}$.

Example 2.7. Suppose that $G=\boldsymbol{Z}_{m}$ ( $m$; odd). Then, for $\sigma=\left[\boldsymbol{Z}_{s} ; V\right] \in$ $\operatorname{St}\left(\boldsymbol{Z}_{m}\right)$ with $s \mid m$, we have

$$
\theta_{\sigma}=(m / s)^{-1}\left\{\chi_{\sigma}+\sum_{s<t \leq m, s|t| m} \varphi(t / s)\left(\sum_{\tau \in \mathscr{S}_{\boldsymbol{Z}_{t}(\sigma)}} \chi_{\tau}\right)\right\}
$$

because $n_{\boldsymbol{Z}_{s}}\left(\boldsymbol{Z}_{t}\right)=\varphi(t / s)$ by definition. The set $\left\{\theta_{\sigma} ;|\sigma| \leq 2 n\right\}$ provides a basis for $\mathscr{T}_{2 n}^{\boldsymbol{Z}_{m}}$.

COROLlary 2.8. Let $H$ be a subgroup of $G$. Then we have

$$
\sum_{H \subseteq K \subseteq G} n_{H}(K) \chi\left(M^{K}\right) \equiv 0 \quad(\bmod |G / H|)
$$

for any G-manifold $M$. In particular, if $H=\{1\}$, then

$$
\sum_{K \subseteq G} n_{\{1\}}(K) \chi\left(M^{K}\right) \equiv 0 \quad(\bmod |G|)
$$

(cf. [6; Corollary 5.19]).

Proof. Consider a sum $\sum_{\sigma} \theta_{\sigma}(M)$ summing over all $\sigma$ with $H$ as an isotropy subgroup. Then it follows from Example 2.5 and Theorem 2.6 that

$$
\begin{aligned}
\sum_{\sigma} \theta_{\sigma}(M) & =|G / H|^{-1}\left\{\chi\left(M^{H}\right)+\sum_{H \subset K \subseteq G} n_{H}(K) \chi\left(M^{K}\right)\right\} \\
& =|G / H|^{-1} \sum_{H \subseteq K \subseteq G} n_{H}(K) \chi\left(M^{K}\right),
\end{aligned}
$$

which is an integer. This gives us the congruence.

\section{3. $G$-fiberings over the circle}

In this section, a $G$-SK invariant is considered to take values in $Z_{2}=\{0,1\}$. If $m$-dimensional $G$-manifolds $M$ and $M^{\prime}$ are $G$-cobordant in the usual sense, then we write $M \stackrel{C}{\sim} M^{\prime}$.

Lemma 3.1 (cf. [5; Lemma 1.9] and [7; Corollary 2.3.2]). Let $M$ and $M^{\prime}$ be m-dimensional $G$-manifolds. 
(i) If $M \sim M^{\prime}$ (SK equivalence), then there is a G-manifold $P$ which fibers equivariantly over the circle $S^{1}$ with the trivial action of $G$ such that $M \stackrel{C}{\sim} M^{\prime}+P$.

(ii) If $M \stackrel{C}{\sim} M^{\prime}$, then $M \sim M^{\prime}+Q$, where

$$
Q=\sum a\left(H, U_{1}, U_{2}\right) \cdot G \times_{H}\left(S\left(U_{1}\right) \times S\left(U_{2}\right)\right)+\sum b(H, U) \cdot G \times_{H} S(U)
$$

for some integers $a\left(H, U_{1}, U_{2}\right)$ and $b(H, U)$. Here, the first sum is taken over all subgroups $H \subseteq G$ and all $H$-modules $U_{i}$ satisfying that $\left(U_{1}\right)^{H}=\{0\}$ such that $\operatorname{dim}\left(U_{1}\right)+\operatorname{dim}\left(U_{2}\right)=m+2$, while the second sum is taken over all $H$ and all $H$-modules $U$ such that $\operatorname{dim}(U)=m+1$.

The relations $\sim$ and $\stackrel{C}{\sim}$ are commutative with each other, i.e. given $M$ and $M^{\prime}$, the following (i) and (ii) are equivalent: (i) there is a $G$-manifold $A$ such that $M \sim A \stackrel{C}{\sim} M^{\prime}$. (ii) there is a $G$-manifold $B$ such that $M \stackrel{C}{\sim} B \sim M^{\prime}$ (cf. [3; Lemma 4.2]).

Definition 3.2. If such an $A$ (or $B$ ) exists, then $M$ and $M^{\prime}$ are said to be $G$-SK equivalent.

We note that $G-\overline{S K}$ equivalence is an equivalence relation by the above commutativity.

Definition 3.3 (cf. [5; Chapter 1]). Let $\overline{S K}_{m}^{G}$ be $\mathscr{M}_{m}^{G}$ factored by the $G-\overline{S K}$ equivalence. In other words, $\overline{S K_{m}^{G}}$ is $S K_{m}^{G}$ factored by the relation $\stackrel{C}{\sim}$.

Let $I_{m}^{G}$ be the kernel of the natural surjection $i_{*}: S K_{m}^{G} \rightarrow \overline{S K}_{m}^{G}$, that is the subgroup of $S K_{m}^{G}$ generated by all elements $[M]-\left[M^{\prime}\right]$ such that $\{M\}=\left\{M^{\prime}\right\}$ in $\mathfrak{R}_{m}^{G}$. Note that $\chi(x)$ is even for any $x \in I_{m}^{G}$ because so is $\chi(M)-\chi\left(M^{\prime}\right)$ (cf. [1; Section 1]).

LEMMA 3.4. $I_{2 n}^{G}=2 S K_{2 n}^{G}$ and $I_{2 n+1}^{G}=\{0\}$.

Proof. In case $m=2 n$, it is sufficient to show that $I_{2 n}^{G} \subseteq 2 S K_{2 n}^{G}$. Take an element $x=[M]-\left[M^{\prime}\right] \in I_{2 n}^{G}$, then $x$ is expressed as

$$
x=\sum a\left(H, U_{1}, U_{2}\right)\left[G \times_{H}\left(S\left(U_{1}\right) \times S\left(U_{2}\right)\right)\right]+\sum b(H, U)\left[G \times_{H} S(U)\right]
$$

by Lemma 3.1 (ii). First, note that $\operatorname{dim} S\left(U_{1}\right)$ is odd by the condition $\left(U_{1}\right)^{H}=\{0\}$. This implies that the first sum of the right-hand side vanishes since $\left[S\left(U_{1}\right)\right]=0$ in $S K_{*}^{H}$ (cf. Proposition 1.4). On the other hand, since $U=\boldsymbol{R}^{2 k+1} \times V$ for some slice type $\sigma=[H ; V](2 k+|\sigma|=2 n)$, we have that 
$\left[G \times_{H} S(U)\right]=2 \alpha^{k} y[\sigma]$ by Lemma 1.7 (i) and Example 2.3. Hence $x \in 2 S K_{2 n}^{G}$. Finally, $I_{2 n+1}^{G}=\{0\}$ since so is $S K_{2 n+1}^{G}$.

From the above, there exists an isomorphism $\overline{S K}_{m}^{G} \cong S K_{m}^{G} / 2 S K_{m}^{G}$. The following theorem is therefore immediate by Proposition 1.4.

THeOREM 3.5. $\overline{S K}_{2 n}^{G}$ is a $Z_{2}$-module with basis $\left\{\alpha^{n-|\sigma| / 2} y[\sigma] ;|\sigma| \leq 2 n\right\} . \quad$ On the other hand, $\overline{S K}_{2 n+1}^{G}=\{0\}$.

Definition 3.6. Let $T: \mathscr{M}_{m}^{G} \rightarrow \boldsymbol{Z}_{2}$ be an additive map. We say that $T$ is a $G-\overline{S K}$ invariant if $T(M)=T\left(M^{\prime}\right)$ for any $M$ and $M^{\prime} \in \mathscr{M}_{m}^{G}$ such that they are $G-\overline{S K}$ equivalent. A $G-\overline{S K}$ invariant $T$ induces a homomorphism $T: \overline{S K}_{m}^{G} \rightarrow \boldsymbol{Z}_{2}$.

Example 3.7. Assume the $M_{C}$ and $M^{\prime}$ are $G-\overline{S K}$ equivalent, i.e. there is a $G$-manifold $A$ such that $M \sim A \stackrel{C}{\sim} M^{\prime}$, then we have $M_{\sigma} \sim A_{\sigma} \stackrel{{ }^{C}}{\sim} M_{\sigma}^{\prime}$ for any $\sigma \in S t(G)$. This means that $M_{\sigma}$ and $M_{\sigma}^{\prime}$ are also $G-\overline{S K}$ equivalent. Thus, $\chi_{\sigma}(\bmod 2)$ defined by $\chi_{\sigma}(M)=\chi\left(M_{\sigma}\right)$ reduced modulo 2 is a $G-\overline{S K}$ invariant.

THeOREM 3.8. Let $\overline{\mathscr{T}}_{m}^{G}$ be the set of all $G-\overline{S K}$ invariants $T: \overline{S K}_{m}^{G} \rightarrow \boldsymbol{Z}_{2}$. Then $\overline{\mathscr{T}}_{2 n}^{G}$ is a $\boldsymbol{Z}_{2}$-module with basis $\left\{\theta_{\sigma}(\bmod 2) ;|\sigma| \leq 2 n\right\}$. On the other hand, $\overline{\mathscr{T}}_{2 n+1}^{G}=\{0\}$.

Proof. The isomorphism in (2.6.3) induces a map

$$
\oplus_{\sigma} \theta_{\sigma}(\bmod 2): S K_{2 n}^{G} \stackrel{\oplus \theta_{\sigma}}{=} \oplus_{\sigma} \boldsymbol{Z} \stackrel{i}{\rightarrow} \oplus_{\sigma} \boldsymbol{Z}_{2},
$$

where the sums are taken over all $\sigma$ with $|\sigma| \leq 2 n$ and $i: Z \rightarrow Z_{2}$ is the natural surjection. Since the kernel of this map is $2 S K_{2 n}^{G}=I_{2 n}^{G}$ by Lemma 3.4, the map $\oplus_{\sigma} \theta_{\sigma}(\bmod 2)$ induces the isomorphism $\overline{S K}_{2 n}^{G} \cong \oplus_{\sigma} \boldsymbol{Z}_{2}$. This verifies that the set $\left\{\theta_{\sigma}(\bmod 2) ;|\sigma| \leq 2 n\right\}$ provides a basis for $\overline{\mathscr{T}}_{2 n}^{G}$. If $m=2 n+1$, then $\overline{\mathscr{T}}_{2 n+1}^{G}$ vanishes because so does $\overline{S K}_{2 n+1}^{G}$.

Let $F_{m}^{G}$ be the kernel of the surjection $j_{*}: \mathfrak{R}_{m}^{G} \rightarrow \overline{S K}_{m}^{G}$, that is the subgroup of $\mathfrak{N}_{m}^{G}$ generated by all classes of the form $\{M\}+\left\{M^{\prime}\right\}$ such that $[M]=\left[M^{\prime}\right]$ in $S K_{m}^{G}$. Let us consider the class $\beta$ which has a representative $M^{\prime}$ fibered equivariantly over the circle $S^{1}$ with a fiber $F$ such that the action of $G$ takes place within $F$. Then $M^{\prime} \sim S^{1} \times F \sim \emptyset$ and $\beta \in F_{m}^{G}$ (cf. [7; Theorem 2.4.1 (i) and (ii)]). It follows from Lemma 3.1 (i) that $F_{m}^{G}$ is precisely generated by all these classes $\beta$.

Remark 3.9. Note that $F_{0}^{G}=\{0\}$. On the other hand, we have that $F_{2 n+1}^{G}=\mathfrak{N}_{2 n+1}^{G}$ because $\overline{S K}_{2 n+1}^{G}=\{0\}$. We can explain this from another point of view as follows. We see that $\mathfrak{N}_{*}^{G}$ is multiplicatively generated over the cobordism ring $\mathfrak{N}_{*}$ by some even-dimensinal $G$-manifolds (cf. [7; Theorem 4.1.1]). 
Hence, if $\operatorname{dim}(M)=2 n+1$, odd, then $\{M\}=\sum_{j} a_{j} L_{j}$, where $a_{j} \in \mathfrak{N}_{*}$ with $\operatorname{dim}\left(a_{j}\right)$, odd and $L_{j} \in \mathfrak{P}_{*}^{G}$ with $\operatorname{dim}\left(L_{j}\right)$, even. Since $\chi\left(a_{j}\right)=0$, we see that each $a_{j}$ has a representative which fibers over the circle (cf. [1; Section 1]). This implies that $\{M\} \in F_{2 n+1}^{G}$ and hence $F_{2 n+1}^{G}=\mathfrak{N}_{2 n+1}^{G}$.

Now we consider a condition that a class $\{M\}$ belongs to $F_{2 n}^{G}$. Given $\{M\} \in F_{2 n}^{G}$, let $M^{\prime}$ be a $G$-manifold such that $M \stackrel{C}{\sim} M^{\prime}$ and it fibers equivariantly over $S^{1}$ with a fiber $F$. Then, for any $\sigma \in S t(G)$ we have that $M_{\sigma} \stackrel{C}{\sim} M_{\sigma}^{\prime}$ which also fibers equivariantly over $S^{1}$ with the fiber $F_{\sigma}$. Hence a necessary condition for $\{M\} \in F_{2 n}^{G}$ is that $\chi\left(M_{\sigma}\right) \equiv 0(\bmod 2)$ for any $\sigma$. We have the following theorem by Theorem 3.8 .

Theorem 3.10. Let $M$ be a 2n-dimensional G-manifold. Then $\{M\} \in F_{2 n}^{G}$ if and only if $\theta_{\sigma}(M) \equiv 0(\bmod 2)$ for any slice types $\sigma \in S t(G)$ with $|\sigma| \leq 2 n$.

The following corollary is immediate by Corollary 2.8 .

Corollary 3.11. A necessary condition for a class $\{M\} \in F_{2 n}^{G}$ is that the following congruence

$$
\chi\left(M^{H}\right)+\sum_{H \subset K \subseteq G} n_{H}(K) \chi\left(M^{K}\right) \equiv 0 \quad(\bmod 2 \cdot|G / H|)
$$

holds for any subgroup $H$ of $G$.

Proposition 3.12. Let $G=Z_{p^{r}}\left(p\right.$; odd prime). Then $\{M\} \in F_{2 n}^{G}$ if and only if

$$
\chi\left(M_{\sigma}\right) \equiv \sum_{\lambda \in \mathscr{C}_{s+1}(\sigma)} \chi\left(M_{\lambda}\right) \quad\left(\bmod 2 p^{r-s}\right)
$$

for any $\sigma=\left[\boldsymbol{Z}_{p^{s}} ; V\right] \in S t(G)$ with $|\sigma| \leq 2 n(0 \leq s \leq r)$, where $\mathscr{S}_{s+1}(\sigma)=\mathscr{S}_{\boldsymbol{Z}_{p^{s+1}}}(\sigma)$ and $\mathscr{S}_{r+1}(\sigma)=\emptyset$.

Proof. By Theorem 3.10, in order that $\{M\} \in F_{2 n}^{G}$, a necessary and sufficient condition is that

$$
p^{r-s} \theta_{\sigma}(M)=\chi_{\sigma}+\sum_{s<t \leq r}\left(p^{t-s}-p^{t-s-1}\right)\left(\sum_{\tau \in \mathscr{T}_{t}(\sigma)} \chi_{\tau}\right) \equiv 0 \quad\left(\bmod 2 p^{r-s}\right)
$$

for any $\sigma=\left[\boldsymbol{Z}_{p^{s}}, V\right] \in \operatorname{St}(G)(0 \leq s \leq r)$, where $\varphi\left(p^{t-s}\right)=p^{t-s}-p^{t-s-1}$ in Example 2.7 and an integer $\chi\left(M_{v}\right)$ is simply written as $\chi_{v}$. We define an integer $h_{v}(M)$ for $v=\left[\boldsymbol{Z}_{p^{t}} ; V\right]$ by

$$
h_{v}(M)=\chi_{v}-\sum_{\omega \in \mathscr{T}_{t+1}(v)} \chi_{\omega}
$$


Since $\mathscr{S}_{s+2}(\sigma)$ is decomposed as $\mathscr{S}_{s+2}(\sigma)=\sum_{\lambda \in \mathscr{S}_{s+1}(\sigma)} \mathscr{S}_{s+2}(\lambda)$ and so on, the righthand side of the congruence (3.12.2) is expressed by the sum of these $h_{v}(M)$ as

$$
\begin{aligned}
\left(\chi_{\sigma}\right. & \left.-\sum_{\lambda \in \mathscr{S}_{s+1}(\sigma)} \chi_{\lambda}\right)+p\left(\sum_{\lambda \in \mathscr{S}_{s+1}(\sigma)}\left(\chi_{\lambda}-\sum_{\mu \in \mathscr{S}_{s+2}(\lambda)} \chi_{\mu}\right)\right) \\
& +p^{2}\left(\sum_{\mu \in \mathscr{S}_{s+2}(\lambda)}\left(\chi_{\mu}-\sum_{\xi \in \mathscr{S}_{s+3}(\mu)} \chi_{\xi}\right)\right) \\
& +\cdots+p^{r-s} \sum_{\tau \in \mathscr{S}_{r}(\rho)} \chi_{\tau} \equiv 0\left(\bmod 2 p^{r-s}\right) .
\end{aligned}
$$

If $\tau=\left[\boldsymbol{Z}_{p^{r}} ; V\right]$, then the above congruence (when $\left.\sigma=\tau\right)$ implies that $h_{\tau}(M)=$ $\chi_{\tau} \equiv 0(\bmod 2)$. We assume that $h_{v}(M) \equiv 0\left(\bmod 2 p^{r-t}\right)$ for any $v=\left[\boldsymbol{Z}_{p^{t}} ; V\right]$ $(s<t \leq r)$. Then, by induction, it follows from (3.12.3) that $h_{\sigma}(M)=\chi_{\sigma}-$ $\sum_{\lambda \in \mathscr{S}_{s+1}(\sigma)} \chi_{\lambda} \equiv 0\left(\bmod 2 p^{r-s}\right)$ for $\sigma=\left[\boldsymbol{Z}_{p^{s}} ; V\right] . \quad$ Therefore the congruences $(3.12 .1)$ are obtained. Conversely, let $M$ satisfy $(3.12 .1)$, that is $h_{\sigma}(M) \equiv 0\left(\bmod 2 p^{r-s}\right)$ for any $\sigma=\left[\boldsymbol{Z}_{p s} ; V\right]$. Taking these in the left-hand side of (3.12.3), we have that $\theta_{\sigma}(M) \equiv 0(\bmod 2)$. Thus $\{M\} \in F_{2 n}^{G}$.

COROllary 3.13. Let $G=\boldsymbol{Z}_{p^{r}}$ ( $p$; odd prime). A necessary condition for a class $\{M\} \in F_{2 n}^{G}$ is that the following congruences

$$
\chi\left(M^{Z_{p} s}\right) \equiv \chi\left(M^{Z_{p^{s+1}}}\right) \quad\left(\bmod 2 p^{r-s}\right) \quad(0 \leq s \leq r)
$$

hold, where $\chi\left(M^{Z_{p^{r+1}}}\right)$ is regarded as zero.

Example 3.14. Finally we give a non-zero element of $F_{2 n}^{G}$ in case $G=\boldsymbol{Z}_{7}$. The non-trivial irreducible $G$-modules are $V_{k}=C$ with a generator of $G$ acting by multiplication by $\exp (2 \pi i k / 7)(1 \leq k \leq 3)$. Let $\eta_{j}$ denote the canonical complex line bundle over $\boldsymbol{C} P^{j}$ and $\eta_{j k}=\eta_{j} \otimes_{C} V_{k}$ the $G$-vector bundle over $C P^{j}$ given by the tensor product of $\eta_{j}$ (with the trivial $G$-action) and the trivial vector bundle $V_{k} \times C P^{j}$. For convenience, we denote $\eta_{0 k}=V_{k}$ and $\eta_{1 k}=V_{k}$. Now consider a $G$-manifold $N=\boldsymbol{C P}\left(\boldsymbol{C}^{s} \times\left(V_{1}\right)^{t}\left(V_{2}\right)^{t}\left(V_{3}\right)^{s}\right)$, the associated complex projective space of a product of $G$-vector bundles $v_{N}=C^{s} \times\left(\underline{V_{1}}\right)^{t}\left(\underline{V_{2}}\right)^{t}\left(\underline{V_{3}}\right)^{s}$ over $B_{N}=* \times$ $\left(\boldsymbol{C} P^{1}\right)^{t}\left(\boldsymbol{C} P^{1}\right)^{t}\left(\boldsymbol{C} P^{1}\right)^{s}=\left(C P^{1}\right)^{2 t+s}(s, t$; odd with $s<t$ and $*=\{p t\}$, the onepoint set). We first show that a class $\{N\}$ is a non-zero element in $\mathfrak{N}_{2 n}^{G}$, where $n=3 s+4 t-1$. For each $\sigma \in S t(G)$, a $G$-vector bundle $v$ is said to be of type $\sigma$ if the subset $\left\{x \in v ; \sigma_{x}=\sigma\right\}$ is precisely its base space $B$. Let $\mathfrak{N}_{*}^{G}[\sigma]$ denote the bundle bordism group of all $G$-vector bundles of type $\sigma$. Given a $G$-manifold $M$, the normal bundle $v$ over the fixed point set $F^{G}$ is the direct sum of those $v_{\sigma}$ (of type $\sigma$ ) over $M_{\sigma}$, where the sum is taken over all $\sigma$ with $G$ as an isotropy subgroup (cf. Remark 2.2). Hence there is a well-defined homomorphism $v_{*}$ : $\mathfrak{N}_{*}^{G} \rightarrow \sum_{\sigma} \mathfrak{N}_{*}^{G}[\sigma]$ given by $v_{*}(\{M\})=\sum_{\sigma}\left\{v_{\sigma}\right\}$. For our element $\{N\}$, we have $v_{*}(\{N\})=\sum_{1 \leq i \leq 4}\left\{v_{i}\right\}$, where each $v_{i}$ is as follows: 


$$
\begin{aligned}
v_{1} & =\boldsymbol{C} P^{s-1} \cdot\left(\underline{V_{1}}\right)^{t}\left(\underline{V_{2}}\right)^{t}\left(\underline{V_{3}}\right)^{s} \rightarrow B_{1} \\
& =\boldsymbol{C} P\left(\boldsymbol{C}^{s} \times\{0\}\{0\}\{0\}\right)=\boldsymbol{C} P^{s-1} \cdot\left(\boldsymbol{C} P^{1}\right)^{2 t+s}, \\
v_{2} & =\boldsymbol{C} P\left(\left(\underline{V_{1}}\right)^{t}\right) \cdot\left(V_{1}\right)^{s}\left(\underline{V_{1}}\right)^{t}\left(\underline{V_{2}}\right)^{s} \rightarrow B_{2} \\
& =\boldsymbol{C P}\left(\{0\} \times\left(\underline{V_{1}}\right)^{t}\{0\}\{0\}\right)=\boldsymbol{C P}\left(\left(\underline{V_{1}}\right)^{t}\right) \cdot *\left(\boldsymbol{C} P^{1}\right)^{t+s}, \\
v_{3} & =\boldsymbol{C P}\left(\left(\underline{V_{2}}\right)^{t}\right) \cdot\left(V_{2}\right)^{s}\left(\underline{V_{1}}\right)^{t+s} \rightarrow B_{3} \\
& =\boldsymbol{C P}\left(\{0\} \times\{0\}\left(\underline{V_{2}}\right)^{t}\{0\}\right)=\boldsymbol{C} P\left(\left(\underline{V_{2}}\right)^{t}\right) \cdot *\left(\boldsymbol{C} P^{1}\right)^{t+s}, \\
v_{4} & =\boldsymbol{C} P\left(\left(\underline{V_{3}}\right)^{s}\right) \cdot\left(V_{3}\right)^{s}\left(\underline{V_{2}}\right)^{t}\left(\underline{V_{1}}\right)^{t} \rightarrow B_{4} \\
& =\boldsymbol{C} P\left(\{0\} \times\{0\}\{0\}\left(\underline{V_{3}}\right)^{s}\right)=\boldsymbol{C P}\left(\left(\underline{V_{3}}\right)^{s}\right) \cdot *\left(\boldsymbol{C} P^{1}\right)^{2 t} .
\end{aligned}
$$

Let $\sigma=\left[G ; V_{1}^{t} V_{2}^{t} V_{3}^{s}\right]$, then it is known that $\mathfrak{N}_{*}^{G}[\sigma]$ is a free $\mathfrak{A}_{*}$-module generated by the classes of monomials

$$
\eta_{J K L}=\eta_{j(1) 1} \cdots \eta_{j(t) 1} \eta_{k(1) 2} \cdots \eta_{k(t) 2} \eta_{l(1) 3} \cdots \eta_{l(s) 3}
$$

with $j(1) \geq \cdots \geq j(t) \geq 0, k(1) \geq \cdots \geq k(t) \geq 0$ and $l(1) \geq \cdots \geq l(s) \geq 0$ (cf. [7; Lemma 3.4.4 and Theorem 4.1.1]). Let $\operatorname{dim}\left(\eta_{J K L}\right)=s+2 t+\sum j(p)+\sum k(q)+$ $\sum l(r)$ be the complex dimension of the total space. Now go back to the image $v_{*}(\{N\})$. It follows from (3.14.1) that $N_{\sigma}=B_{1}+B_{4}$ and $v_{\sigma}=v_{1}+v_{4}$. From the condition that $s$ and $t$ are odd with $s<t$, the monomial $\left(\underline{V_{1}}\right)^{t}\left(\underline{V_{2}}\right)^{t}\left(\underline{V}_{3}\right)^{s}$ in $v_{1}$ has the dimension $2 s+4 t$, which is higher than that of the monomial in $v_{4}$, and its coefficient $\left\{\boldsymbol{C} P^{s-1}\right\}=\left\{\left(\boldsymbol{R} P^{(s-1) / 2}\right)^{2}\right\} \neq 0$ in $\mathfrak{N}_{*}$ (cf. [8; Lemma 7]). This ensure that $\left\{v_{\sigma}\right\} \neq 0$ in $\mathfrak{N}_{*}^{G}[\sigma]$ and $\{N\} \neq 0$ in $\mathfrak{N}_{*}^{G}$. Next we study an SK class $[N]$. By definition, $N$ is fibered equivariantly over the first $C P^{1}$ of the base space $B_{N}=\left(C P^{1}\right)^{2 t+s}$ with fiber $F=\boldsymbol{C P}\left(\boldsymbol{C}^{s} \times V_{1}\left(\underline{V_{1}}\right)^{t-1}\left(V_{2}\right)^{t}\left(\underline{V_{3}}\right)^{s}\right)$. Hence $N \sim C P^{1} \times F$ (cf. [7; Theorem 2.4.1 (iv)]). Continuing this SK processes on $F$ inductively, we have

$$
N \sim\left(C P^{1}\right)^{2 t+s} \times M,
$$

where $M=C P\left(C^{s} \times V_{1}^{t} V_{2}^{t} V_{3}^{s}\right)$. Now we apply the equality (1.8.1) for $M$. Note that $\sigma_{(3)}=\sigma$ and $\sigma_{(1)}=\sigma_{(2)}=\left[G ; V_{1}^{s+t} V_{2}^{s}\right]$. Then we have that

$$
[N]=\left[\left(\boldsymbol{C} P^{1}\right)^{2 t+s}\right]\left(2 s \alpha^{s-1} y[\sigma]+2 t \alpha^{t-1} y\left[\sigma_{(1)}\right]\right)
$$

in $S K_{2 n}^{G}$. Hence $[N]$ vanishes in $\overline{S K}_{2 n}^{G}$ and $\{N\} \in F_{2 n}^{G}$ by Lemma 3.4. The slice types of $N$ are $\sigma_{0}, \sigma$ and $\sigma_{(1)}$, and $N_{\sigma_{0}}=N, N_{\sigma}=B_{1}+B_{4}$ and $N_{\sigma_{(1)}}=B_{2}+B_{3}$ by (3.14.1). Thus $\chi(N)=2^{s+2 t+1}(s+t), \chi\left(N_{\sigma}\right)=\chi\left(B_{1}\right)+\chi\left(B_{4}\right)=2^{s+2 t+1} s$ and $\chi\left(N_{\sigma_{(1)}}\right)=\chi\left(B_{2}\right)+\chi\left(B_{3}\right)=2^{s+2 t+1}$ t. These imply that $\chi(N)=\chi\left(N_{\sigma}\right)+\chi\left(N_{\sigma_{(1)}}\right)=$ $\chi\left(N^{G}\right), \chi\left(N_{\sigma}\right) \equiv 0(\bmod 2)$ and $\chi\left(N_{\sigma_{(1)}}\right) \equiv 0(\bmod 2)$, from which the congruences (3.12.1) are obviously satisfied. 


\section{REFERENCES}

[ 1 ] P. E. Conner and E. E. Floyd, Fibring within a cobordism class, Michigan Math. J., 12 (1965), 33-47.

[2] T. HARA, Equivariant SK invariants on $\boldsymbol{Z}_{2^{r}}$ manifolds with boundary, Kyushu J. Math., 53 (1999), 17-36.

[3] T. Hara, SK invariants on closed $\boldsymbol{Z}_{2^{r}}$ manifolds, Kyushu J. Math., 54 (2000), 307-331.

[ 4 ] T. Hara, Equivariant cutting and pasting of G manifolds, Tokyo J. Math., 23 (2000), 69-85.

[5] U. Karras, M. Kreck, W. D. Neumann and E. Ossa, Cutting and Pasting of Manifolds; SK-Groups, Mathematics Lecture Series 1, Publish or Perish, Boston, 1973.

[6] K. Kawakubo, The Theory of Transformation Groups, The Clarendon Press, Oxford University Press, New York, 1991.

[7] C. Kosniowski, Actions of Finite Abelian Groups, Research Notes in Mathematics 18, Pitman, London, 1978.

[ 8 ] C. T. C. WALL, Determination of the cobordism ring, Ann. of Math. (2), 72 (1960), 292-311.

Department of Mathematics

FACULTY OF ENGINEERING

TOKYo University OF SCIENCE

1-3 Kagurazaka, Shinjuku-Ku,

162-8601 JAPAN

e-mail: hara@rs.kagu.tus.ac.jp 